\title{
Slightly Regular Measures and Measureable Sets
}

\author{
James Camacho Jr. \\ New Jersey City University, 2039 Kennedy Boulevard, Jersey City, NJ 07305, USA \\ Correspondence should be addressed to James Camacho Jr.; jcamacho@njcu.edu
}

Received 13 July 2016; Accepted 30 August 2016

Academic Editor: Basil K. Papadopoulos

Copyright (C) 2016 James Camacho Jr. This is an open access article distributed under the Creative Commons Attribution License, which permits unrestricted use, distribution, and reproduction in any medium, provided the original work is properly cited.

Outer and inner measures of a measure $\mu$ are defined and used to prove results involving them on a lattice $\ell$ and its complement $\ell^{\prime}$. The results concern slightly regular measures and sets such as $S_{\mu}$ which is the collection of $\mu$-measureable sets.

\section{Introduction}

Let $X$ be an nonempty set and $\ell$ a lattice of subsets of $X$ such that $\phi$ and $X$ belong to $\ell$. Outer and inner measures of a measure are used to generate results on a lattice as in [1], giving more results for slightly regular measures which were studied in $[2,3]$. In Section 2, these outer and inner measures along with slightly regular measures are introduced with relevant notations, definitions, and properties. Some results are presented in Section 3 for a measure on a lattice. Here, the results concern mostly slightly regular measures and measureable sets.

\section{Background and Notations}

We follow standard notation and definitions, according to [1-9]. Let $X$ be a nonempty set and $\ell$ a lattice of subsets of $X$ such that $\phi$ and $X$ belong to $\ell$. $A(\ell)$ denotes the algebra generated by $\ell$, and $M(\ell)$ denotes those nontrivial, finitely additive measures on $A(\ell) . M_{R}(\ell)$ denotes those elements of $M(\ell)$ that are $\ell$-regular. Note $\mu$ is $\ell$-regular if we have $\mu(E)=\sup \{\mu(L) \mid L \subset E, E \in A(\ell), L \in \ell\}$. Let $M_{\sigma}(\ell)$ be the set of those elements of $M(\ell)$ that are $\sigma$-smooth on $\ell$. Note $\mu$ is $\sigma$-smooth on $\ell$ if $L_{n}, L \in \ell$ and $L_{n} \downarrow \phi$ implies $\mu\left(L_{n}\right) \rightarrow 0 . M \sigma(\ell)$ is the class of the strongly $\sigma$-smooth measures on $\ell$. $\mu$ is strongly $\sigma$-smooth on $\ell$ if $L_{n} \in \ell$ and $L_{n} \downarrow L$ implies $\mu(L)=\inf \mu\left(L_{n}\right)=\lim \mu\left(L_{n}\right) . M^{\sigma}(\ell)$ are those elements of $M(\ell)$ that are $\sigma$-smooth on $A(\ell)$ and are, equivalently, countably additive. Note that $\mu$ is $\sigma$-smooth on $A(\ell)$ if $L_{n}, L \in A(\ell)$ and $L_{n} \downarrow \phi$ imply $\mu\left(L_{n}\right) \rightarrow 0$. We say that $\ell$ is a $\delta$-lattice if $\bigcap_{i} L_{i} \in \ell$ whenever $L_{i} \in \ell$.
For $E \subset X$, we define two outer measures: for $\mu \in M(\ell)$, we have $\mu^{\prime}(E)=\inf \left\{\mu\left(L^{\prime}\right) \mid E \subset L^{\prime}, L \in \ell\right\}$, and for $\mu \epsilon$ $M_{\sigma}(\ell)$ we have

$$
\mu^{\prime \prime}(E)=\inf \left\{\sum_{i} \mu\left(L_{i}^{\prime}\right) \mid E \subset \bigcup_{i} L_{i}^{\prime}, L_{i} \in \ell\right\} .
$$

We have

$$
\begin{aligned}
\mu & \leq \mu^{\prime}(\ell), \\
\mu & =\mu^{\prime}\left(\ell^{\prime}\right) \mu=\mu^{\prime}\left(\ell^{\prime}\right), \\
\mu^{\prime \prime} & \leq \mu\left(\ell^{\prime}\right), \\
\mu^{\prime \prime} & \leq \mu^{\prime} .
\end{aligned}
$$

Note if $\mu \in M_{\sigma}(\ell)$, we have $\mu \leq \mu^{\prime}(\ell)$, for $\mu \in M \sigma(\ell)$; then, $\mu^{\prime}=\mu\left(\ell^{\prime}\right)$, and if $\mu \in M_{R}^{\sigma}(\ell)$, then $\mu^{\prime}=\mu(\ell)$. We also have for our two outer measures $\mu \in M_{w}(\ell) \subseteq M(\ell)$ if $\mu\left(L^{\prime}\right)=$ $\sup \left\{\mu^{\prime}(B) \mid B \subseteq L^{\prime}, B \in \ell\right\}$ for $L \in \ell$ and $\mu \in M_{v}(\ell) \subseteq M_{\sigma}(\ell)$ if $\mu\left(L^{\prime}\right)=\sup \left\{\mu^{\prime \prime}(B) \mid B \subseteq L^{\prime}, B \in \ell\right\}$ for $L \in \ell . M_{w}(\ell)$ is the set of all weakly regular measures on $A(\ell)$, and $M_{v}(\ell)$ is the set of all vaguely regular measures on $A(\ell)$, and also $\mu \in M_{S}(\ell) \subseteq M(\ell)$ if $\bigcap_{i} L_{i}=L$, where $L, L_{i} \in \ell$; then, $\mu(L)=$ $\inf \mu\left(L_{i}\right)$.

Note. It is not difficult to show that $M_{R}^{\sigma}(\ell) \subset M_{S}(\ell) \subset$ $M^{\sigma}(\ell) \subset M \sigma(\ell) \subset M_{\sigma}(\ell)$. The proof of $M_{S}(\ell) \subset M_{\sigma}(\ell)$ is as follows. 
If $L_{i} \downarrow \phi$, then, $\bigcap_{i} L_{i}=\phi$, where $L_{i} \in \ell$; then, $\mu(\phi)=$ $\inf \mu\left(L_{i}\right)$ as $\mu \in M_{S}(\ell)$. Therefore, $\mu(\phi)+\epsilon>\mu\left(L_{i}\right)$, where $\epsilon>0$. Since $\mu(\phi)=0$, then $\mu\left(L_{i}\right)<\epsilon$ so $\mu \in M_{\sigma}(\ell)$, and the proof of $M_{S}(\ell) \subset M^{\sigma}(\ell)$ is given below.

Given that $\mu$ is a finitely subadditive outer measure, we define the notion that $\mu$ is regular if for every $A \subset X$ there exists $E \in S_{\mu}$ such that $A \subset E$ and $\mu(A)=\mu(E)$. In addition, if $\mu$ is a regular outer measure, $E_{i} \uparrow$, and $E_{i} \subset X$, then $\mu\left(\lim E_{i}\right)=\lim \mu\left(E_{i}\right)$, and if $\mu$ is a regular finitely additive outer measure, we have $E \in S_{\mu}$ if and only if $u(L)+\mu\left(L^{\prime}\right)=$ $u(X)$.

We have if $\mu \in M_{\sigma}(\ell)$ that $\mu \in M_{S}(\ell)$ which implies $\mu^{\prime \prime}=$ $\mu^{\prime}$ on $\ell^{\prime}$ and if $\mu^{\prime \prime}=\mu^{\prime}$ on $\ell^{\prime}$ and $\mu^{\prime \prime}$ is regular then $\mu \in M_{S}(\ell)$ (see [3]).

We now define two more set functions.

Let $\mu \in M(\ell)$. For any $E \subseteq X$, we have $\mu_{i}(E)=\sup \{\mu(L) \mid$ $L \subset E, L \in \ell\}$ which is an inner measure.

Let $\mu \in M_{\sigma}(\ell)$. For any $E \subseteq X$, we have $\mu_{j}(E)=\mu(X)-$ $\mu^{\prime \prime}\left(E^{\prime}\right)$.

If $\mu^{\prime \prime}$ is regular, then $\mu_{j}$ is an inner measure.

For $\mu \in M_{\sigma}(\ell)$ and $E \subseteq X$, we get

$$
\begin{aligned}
\mu_{i}(E) & \leq \mu_{j}(E) \\
& \leq \sup \left\{\mu^{\prime \prime}\left(\bigcap_{1}^{\infty} L_{i}\right), \bigcap L_{i} \subset E, \mid L_{i} \in \ell\right\} \\
& \leq \mu^{\prime \prime}(E) \leq \mu^{\prime}(E)(1) .
\end{aligned}
$$

For the proof, see [4].

\section{Results}

The following theorem is proved in [2] using $I_{S}(\ell)$ which is a subset of $M_{S}(\ell)$ consisting of zero-one valued measures.

Theorem 1. If $\mu, \nu \in M_{S}(\ell), \mu \leq \nu(\ell)$, and $\mu(X)=\nu(X)$, then $\mu=v$.

Proof. For $L \in \ell \subseteq X$, we have that $\mu \leq \nu(\ell)$ implies $\nu \leq \mu\left(\ell^{\prime}\right)$ so $\mu\left(L^{\prime}\right) \geq \nu\left(L^{\prime}\right)$. Now, $\mu\left(L^{\prime}\right)+\epsilon \geq \nu\left(L^{\prime}\right)+\epsilon=\nu^{\prime \prime}\left(L^{\prime}\right)+\epsilon$ as $\mu \epsilon$ $M_{S}(\ell)$, which implies $\mu^{\prime \prime}=\mu^{\prime}$ on $\ell^{\prime}$ and $\mu=\mu^{\prime}$ on $\ell^{\prime}$ always. Now $\mu\left(L^{\prime}\right)+\epsilon \geq \nu\left(L^{\prime}\right)+\epsilon=\nu^{\prime \prime}\left(L^{\prime}\right)+\epsilon>\sum \nu\left(L_{n}^{\prime}\right)>v\left(L_{n}^{\prime}\right)$ by the definition of $v^{\prime \prime}$.

Therefore, $\mu\left(L^{\prime}\right)+\epsilon \geq \nu\left(L_{n}^{\prime}\right)$. It follows that $-\mu\left(L^{\prime}\right)-\epsilon \leq$ $-v\left(L_{n}^{\prime}\right)$ and $\mu(X)-\mu\left(L^{\prime}\right)-\epsilon \leq \nu(X)-\nu\left(L_{n}^{\prime}\right)$ as $\mu(X)=\nu(X)$.

Therefore, $\mu(L)-\epsilon \leq \nu\left(L_{n}\right)$ giving us $\mu(L) \geq \nu\left(L_{n}\right)$. This implies that $\mu(L)$ is an upper bound of $\nu\left(L_{n}\right)$. So sup $\nu\left(L_{n}\right) \leq$ $\mu(L)$ and it follows that inf $\mu\left(L_{n}\right) \leq \inf \nu\left(L_{n}\right) \leq \sup v\left(L_{n}\right) \leq$ $\mu(L)$ as $\mu \leq \nu(\ell)$. Using $\mu, v \in M_{S}(\ell)$, we get $\mu(L) \leq \nu(L) \leq$ $\sup \nu\left(L_{n}\right) \leq \mu(L)$ as inf $\mu\left(L_{n}\right)=\mu(L)$ and inf $\nu\left(L_{n}\right)=\nu(L)$. Therefore, $\mu(L) \leq \nu(L) \leq \mu(L)$ giving us $\mu=\nu$.

Theorem 2. If $\mu \in M_{S}(\ell)$ and if there exists $\nu \in M_{R}^{\sigma}(\ell)$ such that $\mu \leq \nu(\ell)$ and $\mu(X)=\nu(X)$, then $\mu=\nu$.

Proof. Since $M_{R}^{\sigma}(\ell) \subset M_{S}(\ell)$, then $\nu \in M_{R}^{\sigma}(\ell)$ implies $v \in$ $M_{S}(\ell)$. Therefore, $\mu=\nu$ by Theorem 1 .
Theorem 3. If $\mu \in M_{S}(\ell), \mu \leq \nu(\ell)$, $\ell$ is a $\delta$-lattice, $\mu(X)=$ $\mu^{\prime}(X)$, and $\nu \in M_{\sigma}(\ell)$, then $\nu \in M_{S}(\ell)$ and therefore $\mu=\nu$.

Proof. If $L_{i} \downarrow L$, then, $\bigcap_{i} L_{i}=L$ and so $L=\bigcap_{i} L_{i} \subseteq L_{i}$. It follows that $\nu(L) \leq \nu\left(L_{i}\right)$ so $v(L)$ is a lower bound of $v\left(L_{i}\right)$. Therefore, $v(L) \leq \inf v\left(L_{i}\right)$. Now inf $v\left(L_{i}\right) \leq \sup v\left(L_{i}\right)$ so $\nu(L) \leq \inf \nu\left(L_{i}\right) \leq \sup v\left(L_{i}\right)$. Now $\mu^{\prime \prime}\left(L^{\prime}\right)+\epsilon>\mu\left(L_{i}^{\prime}\right)$ by definition of $\mu^{\prime \prime}$ so $-\mu^{\prime \prime}\left(L^{\prime}\right)-\epsilon<-\mu\left(L_{i}^{\prime}\right)$, and since $\mu(X)=\mu^{\prime}(X)$ and $\mu^{\prime}=\mu^{\prime \prime}$ on $\ell^{\prime}$, we have $\mu(X)-\mu^{\prime}\left(L^{\prime}\right)-\epsilon<$ $\mu(X)-\mu\left(L_{i}^{\prime}\right)$ giving us $\mu^{\prime}(L)-\epsilon<\mu\left(L_{i}\right)$.

Since $\mu \leq \mu^{\prime}(\ell)$, we have $\mu(L)-\epsilon<\mu^{\prime}(L)-\epsilon<\mu\left(L_{i}\right)$ giving $\mu(L)-\epsilon<\mu\left(L_{i}\right)$ and $\mu(L)-\epsilon<\mu\left(L_{i}\right)<\nu\left(L_{i}\right)$ as $\mu \leq \nu(\ell)$ which implies $\mu(L) \geq \nu\left(L_{i}\right)$ so $\mu(L)$ is an upper bound of $\nu\left(L_{i}\right)$ giving $\mu(L) \geq \sup v\left(L_{i}\right)$. So we have sup $\nu\left(L_{i}\right) \leq \mu(L) \leq \nu(L)$ as $\mu \leq \nu(\ell)$. Remember we showed early in the proof that $v(L) \leq \inf v\left(L_{i}\right) \leq \sup v\left(L_{i}\right)$ and therefore we have $v(L) \leq$ $\inf v\left(L_{i}\right) \leq \sup v\left(L_{i}\right) \leq \mu(L) \leq \nu(L)$ which gives us $\nu(L)=$ $\inf v\left(L_{i}\right)$. So $v \in M_{S}(\ell)$. Therefore, $\mu=v$ by Theorem 1 .

Theorem 4. If $\mu \in M_{S}(\ell) \cap M_{\sigma}(\ell)$, then $\mu=\mu^{\prime \prime}(\ell)$.

Proof. We know since $\mu \in M_{\sigma}(\ell)$, we have $\mu \leq \mu^{\prime}(\ell)$. Let $\epsilon>$ $0, L_{i} \downarrow L$, and $L . L_{i} \in \ell$ so then $\mu^{\prime \prime}\left(L^{\prime}\right)+\epsilon>\sum_{i} \mu\left(L_{i}^{\prime}\right)$, where $L^{\prime} \subset \bigcup_{i} L_{i}^{\prime}$ for $L_{i} \in L$. We have $L=\bigcap\left(L_{i} \cup L\right)$ and $L_{i} \cup L \downarrow L$ as $L_{i} \downarrow L$ and as $\mu \in M_{S}(\ell)$; then, $\mu(L)+\epsilon>\mu\left(L_{N} \cup L\right)$ for a fixed $N$. Now since $\mu \leq \mu^{\prime}(\ell)$, we have $\mu^{\prime}(L)+\epsilon>\mu(L)+$ $\epsilon>\mu\left(L_{N} \cup L\right)$ and by the monotone property of $\mu$, we have $\mu^{\prime}(L)+\epsilon>\mu(L)+\epsilon>\mu\left(L_{N} \cup L\right)>\mu(L)$. So $\mu^{\prime}(\ell) \leq \mu(\ell)$ as $\mu^{\prime}(L)+\epsilon>\mu(L)$ and since $\mu \leq \mu^{\prime}(\ell)$ we get $\mu=\mu^{\prime \prime}(\ell)$.

Note for the next results $S_{\mu^{\prime}}$ is the collection of $\mu^{\prime}$ measureable sets, and $S_{\mu^{\prime \prime}}$ is the collection of $\mu^{\prime \prime}$-measureable sets.

Theorem 5. If $\mu \in M_{S}(\ell) \cap M_{\sigma}(\ell), \ell$ is a $\delta$-lattice, and $\mu(X)=$ $\mu^{\prime}(X)$, then $\ell \subset S_{\mu^{\prime}}$.

Proof. We have $\mu_{j}=\mu^{\prime}=\mu^{\prime \prime}$ on $\ell$; as for $L \in \ell \subseteq X$, we have $\mu_{j}(L)=\mu(X)-\mu^{\prime \prime}\left(L^{\prime}\right)$. Since $\mu \in M_{S}(\ell)$, then $\mu^{\prime \prime}=\mu^{\prime}$ on $\ell^{\prime}$ so $\mu_{j}(L)=\mu(X)-\mu^{\prime}\left(L^{\prime}\right)=\mu^{\prime}(L)$ as $\mu^{\prime}\left(L^{\prime}\right)+\mu^{\prime}\left(L^{\prime}\right)=\mu^{\prime}(X)$. Therefore, $\mu_{j}=\mu^{\prime}$ on $\ell$.

From $\mu_{i}(E) \leq \mu_{j}(E) \leq \sup \left\{\mu^{\prime \prime}\left(\bigcap_{1}^{\infty} L_{i}\right), \bigcap L_{i} \subset E, \mid L_{i} \in\right.$ $\ell\} \leq \mu^{\prime \prime}(E) \leq \mu^{\prime}(E)(1)$, we have with $E=L$ that $\mu_{i}(L) \leq$ $\mu_{j}(L) \leq \sup \left\{\mu^{\prime \prime}\left(\bigcap_{1}^{\infty} L_{i}\right), \bigcap L_{i} \subset L, \mid L_{i} \in \ell\right\} \leq \mu^{\prime \prime}(L) \leq$ $\mu^{\prime}(L)$. Therefore, $\mu_{i}(L) \leq \mu_{j}(L) \leq \sup \left\{\mu^{\prime \prime}\left(\bigcap_{1}^{\infty} L_{i}\right), \bigcap L_{i} \subset\right.$ $\left.L, \mid L_{i} \in \ell\right\} \leq \mu^{\prime \prime}(L) \leq \mu^{\prime}(L)=\mu_{j}(L)$ as $\mu_{j}=\mu^{\prime}$ on $\ell$. Therefore, $\mu_{j}=\mu^{\prime}=\mu^{\prime \prime}$ on $\ell$, and using $\mu_{i}(E) \leq \mu_{j}(E) \leq$ $\sup \left\{\mu^{\prime \prime}\left(\bigcap_{1}^{\infty} L_{i}\right), \bigcap L_{i} \subset E, \mid L_{i} \in \ell\right\} \leq \mu^{\prime \prime}(E) \leq \mu^{\prime}(E)(1)$ for $E \in \ell$, we get $\mu^{\prime}(E)=\sup \left\{\mu^{\prime \prime}\left(\bigcap_{1}^{\infty} L_{i}\right), \bigcap L_{i} \subset E, \mid L_{i} \in \ell\right\}$. Also since $\ell$ is a $\delta$-lattice, we get $\bigcap_{1}^{\infty} L_{i}=L \in \ell$ so we get $\mu^{\prime}(E)=\sup \left\{\mu^{\prime \prime}(L), L \subset E, \mid L, E \in \ell\right\}$. Now $\mu=\mu^{\prime \prime}(\ell)$ from Theorem 4 so $\mu^{\prime}(E)=\sup \{\mu(L), L \subset E, \mid L, E \in \ell\}$ and it follows from [3] that $E \in S_{\mu^{\prime}}$. Since $E \in \ell$, we have $\ell \subset S_{\mu^{\prime}}$.

Theorem 6. If $\mu \in M_{S}(\ell) \cap M_{\sigma}(\ell)$, $\ell$ is a $\delta$-lattice, and $\mu(X)=$ $\mu^{\prime}(X)$, then $\ell \subset S_{\mu^{\prime \prime}}$. 
Proof. Since $\mu \in M_{S}(\ell)$ and $\ell$ is a $\delta$-lattice, then $S_{\mu^{\prime}}=S_{\mu^{\prime \prime}}$ from Theorem 4.8 part (c) of [3]. Now since $\mu \in M_{S}(\ell) \cap$ $M_{\sigma}(\ell), \ell$ is a $\delta$-lattice, and $\mu(X)=\mu^{\prime}(X)$, then $\ell \subset S_{\mu^{\prime}}$ from Theorem 5. So since $S_{\mu^{\prime}}=S_{\mu^{\prime \prime}}$, we get $\ell \subset S_{\mu^{\prime \prime}}$.

Theorem 7. If $\mu \in M_{S}(\ell) \cap M_{\sigma}(\ell)$, $\ell$ is a $\delta$-lattice, and $\mu(X)=$ $\mu^{\prime}(X)$, then $\mu \in M^{\sigma}(\ell)$.

Proof. From Theorem 6, we have $\ell \subset S_{\mu^{\prime \prime}}$, so $\mu^{\prime \prime}$ is countably additive on $A(\ell)$. Now since $\mu \in M_{S}(\ell) \cap M_{\sigma}(\ell)$, then $\mu=$ $\mu^{\prime \prime}(\ell)$ from Theorem 4 so $\mu$ is countably additive on $A(\ell)$. Therefore, $\mu \in M^{\sigma}(\ell)$ by definition.

Theorem 8. If $E \in S_{\mu^{\prime}}$, then $\mu^{\prime}=\mu_{i}$.

Proof. From Lemma 4.1 of [3], $E \in S_{\mu^{\prime}}$ if and only if $\mu^{\prime}(E)=$ $\sup \{\mu(L), L \subset E, \mid L, E \in \ell\}$. Now $\mu_{i}(E)=\sup \{\mu(L) \mid L \subset$ $E, L \in \ell\}$; therefore, $\mu^{\prime}=\mu_{i}$.

Theorem 9. If $E \in S_{\mu^{\prime}}$, then $\mu^{\prime}=\mu_{i}=\mu_{j}=\mu^{\prime \prime}$.

Proof. If $E \in S_{\mu^{\prime}}$, then $\mu^{\prime}=\mu_{i}$ from Theorem 8. Now using $\mu_{i}(E) \leq \mu_{j}(E) \leq \sup \left\{\mu^{\prime \prime}\left(\bigcap_{1}^{\infty} L_{i}\right), \bigcap L_{i} \subset E, \mid L_{i} \in \ell\right\} \leq$ $\mu^{\prime \prime}(E) \leq \mu^{\prime}(E)(1)$ for $E \in S_{\mu^{\prime}}$ we get $\mu^{\prime}=\mu_{i}=\mu_{j}=\mu^{\prime \prime}$.

Theorem 10. If $\mu \in M_{S}(\ell) \cap M_{\sigma}(\ell), \mu^{\prime}=\mu_{i}$, and $\ell$ is $a \delta$ lattice, then $E \in S_{\mu^{\prime}}$.

Proof. Since $\mu^{\prime}=\mu_{i}, \mu_{i}(E) \leq \mu_{j}(E) \leq \sup \left\{\mu^{\prime \prime}\left(\bigcap_{1}^{\infty} L_{i}\right), \bigcap L_{i} \subset\right.$ $\left.E, \mid L_{i} \in \ell\right\} \leq \mu^{\prime \prime}(E) \leq \mu^{\prime}(E)(1)$ becomes $\mu^{\prime}(E)=$ $\sup \left\{\mu^{\prime \prime}\left(\bigcap_{1}^{\infty} L_{i}\right), \bigcap L_{i} \subset E, \mid L_{i} \in \ell\right\}$. As $\ell$ is a $\delta$-lattice, we get $\bigcap_{1}^{\infty} L_{i}=L \in \ell$ so we get $\mu^{\prime}(E)=\sup \left\{\mu^{\prime \prime}(L), L \subset E, \mid L \in \ell\right\}$ and since $\mu \in M_{S}(\ell) \cap M_{\sigma}(\ell)$ we have by Theorem 4 that $\mu=\mu^{\prime \prime}(\ell)$ so $\mu^{\prime}(E)=\sup \{\mu(L), L \subset E, \mid L \in \ell\}$, and from [3] this implies $E \in S_{\mu^{\prime}}$.

Theorem 11. If $\mu \in M_{S}(\ell)$ and $\ell$ is a $\delta$-lattice, then $S_{\mu^{\prime \prime}} \cap \ell=$ $\left\{L \in \ell \mid \mu(L)=\mu^{\prime \prime}(L)\right\}$.

Proof. Let $L \in S_{\mu^{\prime \prime}} \cap \ell$; then, $L \in S_{\mu^{\prime}} \cap \ell$ as $S_{\mu^{\prime}}=S_{\mu^{\prime \prime}}$ from [3], and in [3] we have that if $\mu \in M(\ell)$, then $S_{\mu^{\prime}} \cap \ell=\{L \in \ell \mid$ $\left.\mu(L)=\mu^{\prime}(L)\right\}$. So $L \in S_{\mu^{\prime}} \cap \ell$ implies $\mu(L)=\mu^{\prime}(L)$. However, $\mu \in M_{S}(\ell)$, and $\ell$ is a $\delta$-lattice implying $\mu^{\prime \prime}=\mu^{\prime}$ everywhere in [3] so $\mu(L)=\mu^{\prime \prime}(L)$.

Now let $\mu(L)=\mu^{\prime \prime}(L)$ for $L \in \ell$; then, $\mu(L)=\mu^{\prime}(L)$ as $\mu^{\prime \prime}=\mu^{\prime}$ everywhere. Therefore, $L \in S_{\mu^{\prime}} \cap \ell$ and as $S_{\mu^{\prime}}=S_{\mu^{\prime \prime}}$ we have $L \in S_{\mu^{\prime \prime}} \cap \ell$.

We now end with a sort of converse to Theorem 4 .

Theorem 12. If $\mu \in M_{\sigma}(\ell), \mu(X)=\mu^{\prime \prime}(X), \mu=\mu^{\prime \prime}(\ell), \ell \subset S_{\mu}$, and $\mu^{\prime \prime}$ is regular, then $\mu \in M_{S}(\ell)$.

Proof. Now $\mu\left(L^{\prime}\right)=u(X)-u(L)=\mu^{\prime \prime}(X)-\mu^{\prime \prime}(L)$ as $\mu(X)=$ $\mu^{\prime \prime}(X)$ and $\mu=\mu^{\prime \prime}(\ell)$. Therefore, $\mu\left(L^{\prime}\right)=\mu^{\prime \prime}(X)-\mu^{\prime \prime}(L)$ giving us $\mu\left(L^{\prime}\right)=\mu^{\prime \prime}\left(L^{\prime}\right)$ as $\mu^{\prime \prime}$ is regular, so we have $\mu=$ $\mu^{\prime \prime}\left(\ell^{\prime}\right)$. Then, it follows $\mu \in M_{S}(\ell)$ from [3].

\section{Competing Interests}

The author declares that there is no conflict of interests regarding the publication of this paper.

\section{References}

[1] J. Camacho, "Outer and inner lattice measures," The Journal of the Indian Mathematical Society, vol. 80, no. 1-4, pp. 1-11, 2013.

[2] J. Camacho, "On topological properties of certain Wallman spaces,” Journal of Mathematical Sciences, vol. 7, no. 1, pp. 3344, 1996.

[3] P. M. Grassi, "Outer measures and associated lattice properties," International Journal of Mathematics and Mathematical Sciences, vol. 16, no. 4, pp. 687-694, 1993.

[4] P. Grassi, "On subspaces of replete and measure replete spaces," Canadian Mathematical Bulletin, vol. 27, no. 1, pp. 58-64, 1984.

[5] P.-S. Hsu, "Characterizations of outer measures associated with lattice measures," International Journal of Mathematics and Mathematical Sciences, vol. 24, no. 4, pp. 237-249, 2000.

[6] C. Huerta, "Notions of compactness on the lattice and on the point set in terms of measure," Annales des Sciences Mathematiques du Quebec, vol. 13, no. 1, pp. 49-52, 1989.

[7] M. Szeto, "Measure Repleteness and mapping preservations," The Journal of the Indian Mathematical Society, vol. 43, pp. 35$52,1979$.

[8] C. Traina, "On finitely subadditive outer measures and modularity properties," International Journal of Mathematics and Mathematical Sciences, no. 8, pp. 461-474, 2003.

[9] C. D. Vlad, "On compactness of lattices," International Journal of Mathematics and Mathematical Sciences, no. 16, pp. 2565-2573, 2005. 


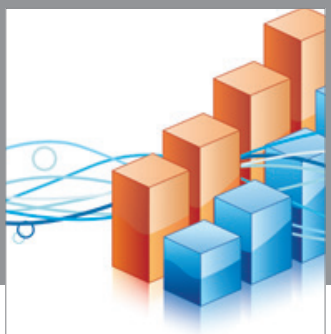

Advances in

Operations Research

vatem alat4

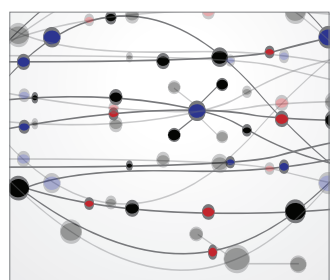

\section{The Scientific} World Journal
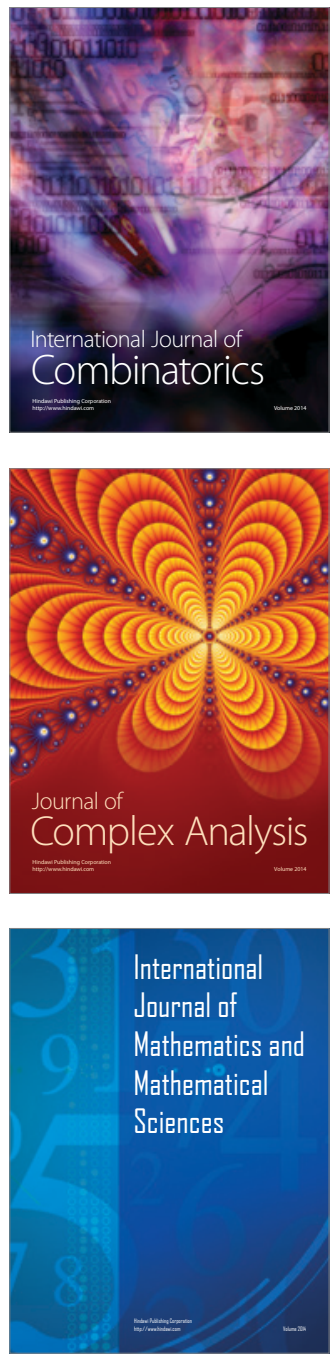
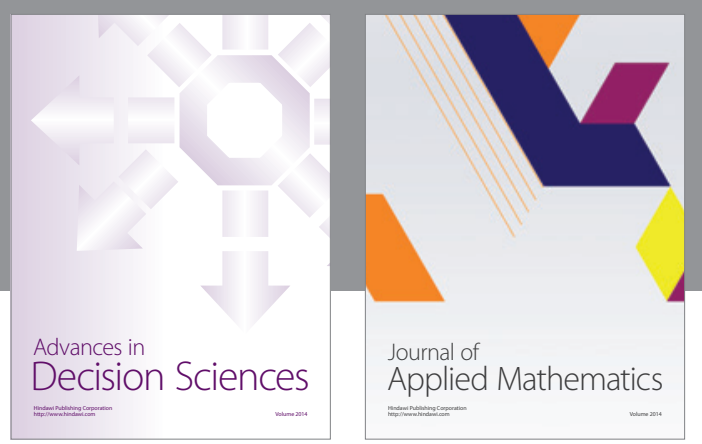

Algebra

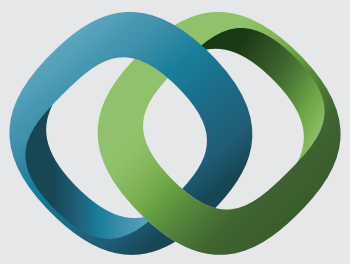

\section{Hindawi}

Submit your manuscripts at

http://www.hindawi.com
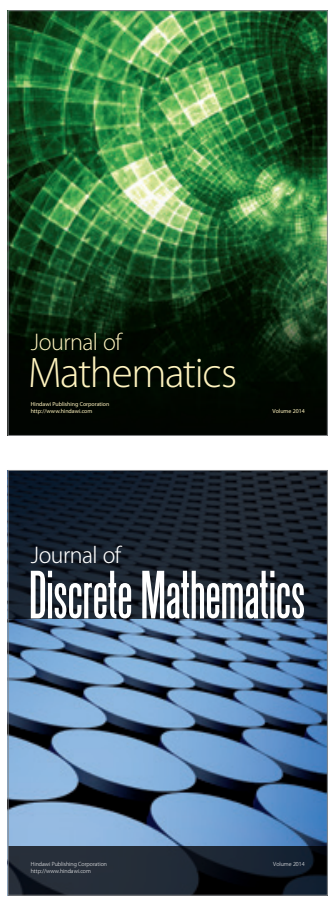

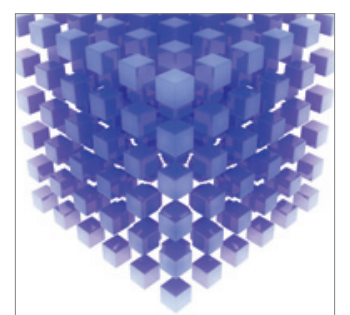

Mathematical Problems in Engineering
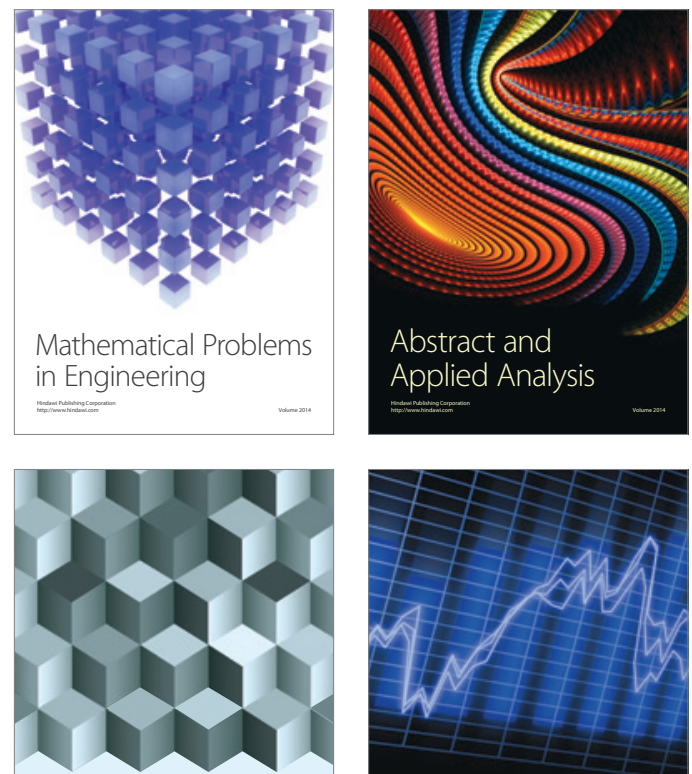

Journal of

Function Spaces

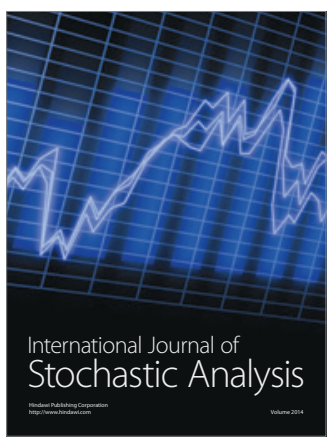

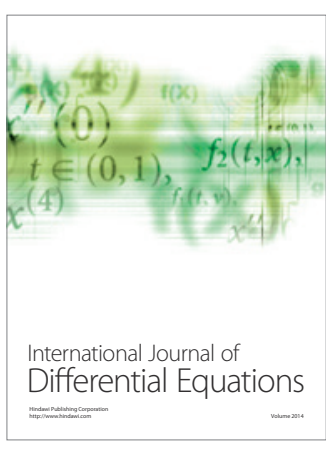
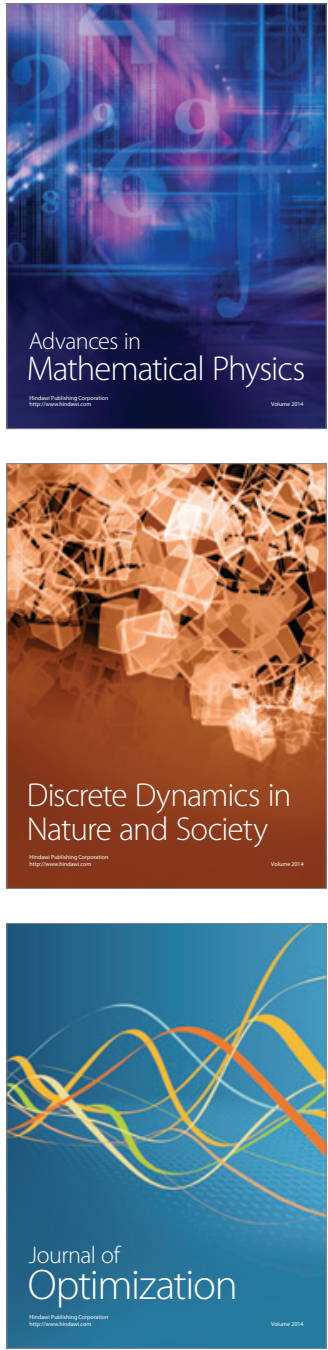